\title{
The morphological characters of the male external genitalia of the European hedgehog (Erinaceus Europaeus)
}

\author{
G. Akbari ${ }^{1}$, M. Babaei ${ }^{1}$, N. Goodarzi \\ ${ }^{1}$ Department of Basic Sciences, Faculty of Veterinary Medicine, University of Tabriz, Tabriz, Iran \\ ${ }^{2}$ Department of Basic Sciences, Faculty of Veterinary Medicine, Razi University, Kermanshah, Iran \\ [Received: 7 June 2017; Accepted: 11 September 2017]
}

\begin{abstract}
This study was conducted to depict anatomical characteristics of the penis of hedgehog. Seven sexually mature male European hedgehogs were used. Following anaesthesia, the animals were scarified with chloroform inhalation. Gross penile characteristics such as length and diameter were thoroughly explored and measured using digital callipers. Tissue samples stained with haematoxylin and eosin and Masson's trichrome for microscopic analysis. The penis of the European hedgehog was composed of a pair of corpus cavernosum penis and the glans penis without corpus spongiosum penis. The urethra at the end of penis, protruded as urethral process, on both sides of which two black nail-like structures, could be observed. The lower part was rounded forming a blind sac (sacculus urethralis) with a median split below the urethra. Microscopically, the penile bulb lacked the corpus spongiosum penis, but, corpus spongiosum glans was seen at the beginning of the free part. In the European hedgehog, entirely stratified squamous epithelium of penile urethra, absence of corpus spongiosum penis around the urethra and bilateral urethral glands are basically different compared with other mammals. This information is expected to contribute to comparative penile morphology as well as for testing phylogenic hypotheses and expanding knowledge about reproductive biology in this animal. (Folia Morphol 2018; 77, 2: 293-300)
\end{abstract}

Key words: corpus spongiosum glans, European hedgehog, morphology, penis, urethra

\section{INTRODUCTION}

Insectivores are phylogenetically deemed to be among the earliest Eutheria. According to reports, their characteristics are closer to those of primates than those of rodents on the basis of, for instance, tooth morphologies or mitochondrial DNA sequences $[11,21,28]$.

As the focus of the present research, the hedgehog refers to any spiny mammal from the subfamily Erinaceinae and the order Eulipotyphla. There are 17 species of this animal in 5 genera. The large population of European hedgehogs (Erinaceus Europaeus) there are in Europe, Africa, Asia, United States [USA], and New Zealand. Pet owners have just devoted a considerable attention to these spiny animals. The popularity of hedgehog in some countries such as USA and Iran is increasing because they are cute and different and, moreover, they are considered low-maintenance pets [42]. A better understanding of their reproductive biology will therefore improve their domesticating process and captive rearing; in addition, it will increase stock levels and intensify production practices. 
As anatomical structures, external genitals are commonly categorised as an appendage. These sex organs are essential for mating and internal fertilisation among lots of mammalian species. It is clinically important to analyse the penis morphologically $[19,41]$, which can enormously contribute to the reassessment of the phylogeny since it uncovers diverse phylogenetic characters hidden in this order $[9,32]$.

The penile anatomy of both domestic $[16,27]$ and laboratory animals $[12,15,18,24,30,35]$ has been delineated. Not much information is however available on the penile anatomy of wild animals and no report exists on the penile anatomy of the European hedgehog.

Thus, the present study attempts to offer a vivid description of the anatomy of the penis of the European hedgehog with the aim of supplying information which is expected to contribute to comparative studies on penile morphology. Moreover, the information will be useful for testing phylogenic hypotheses and expanding knowledge about reproductive biology in this animal.

\section{MATERIALS AND METHODS}

The present study was carried on seven (weight: $783.32 \pm 34.67 \mathrm{~g}$ ) sexually mature male European hedgehogs (Erinaceus Europaeus). They were captured from suburbs of Tabriz, East Azerbaijan, Iran. The animals were fed with insects and given unlimited access to water. All animal procedures conducted in this research were approved by standards of the University of Tabriz on Humane Care and Use of Laboratory Animals, in accordance with the Research Ethics Committee of the Ministry of Health and Medical Education in Iran (adopted on April 17, 2006), based on the Helsinki Protocol (Helsinki, Finland, 1975). Prior to their sacrifice following anaesthesia with chloroform in a closed container, the hedgehogs were individually weighed (weighing scale: Shimadzu AW320, Germany). The carcass of each hedgehog was placed on its back and the fore as well as hind legs were laterally spread. The penile organ was first examined in situ. It was re-examined after the removal. Gross penile characteristics were thoroughly explored and recorded. The length and diameter of the penis were measured utilising digital callipers. Images of the penile structures were taken with a digital camera. Furthermore, the penises were trimmed and fixed in $10 \%$ neutral formalin solution and then embedded in paraffin. Serial $5 \mu \mathrm{m}$ sagittal and vertical sections were stained with haematoxylin and eosin (H\&E) and Masson's trichrome connective tissue stain (MTC). A microscope (model BX51; Olympus, Tokyo, Japan) which was coupled to a digital camera (model
Table 1. Length and diameter of European hedgehog penis

\begin{tabular}{lll}
\hline Total length $[\mathrm{mm}]$ & & $7.17 \pm 0.12$ \\
\hline Length $[\mathrm{mm}]$ & Free part & $2.02 \pm 0.44$ \\
& Body & $3.58 \pm 0.11$ \\
& Root & $1.31 \pm 0.02$ \\
\hline Diameter [mm] & Free part & $0.63 \pm 0.03$ \\
& Body & $0.83 \pm 0.05$ \\
& Root & $1.05 \pm 0.06$ \\
\hline
\end{tabular}

Measurements are expressed as mean \pm standard deviation values.

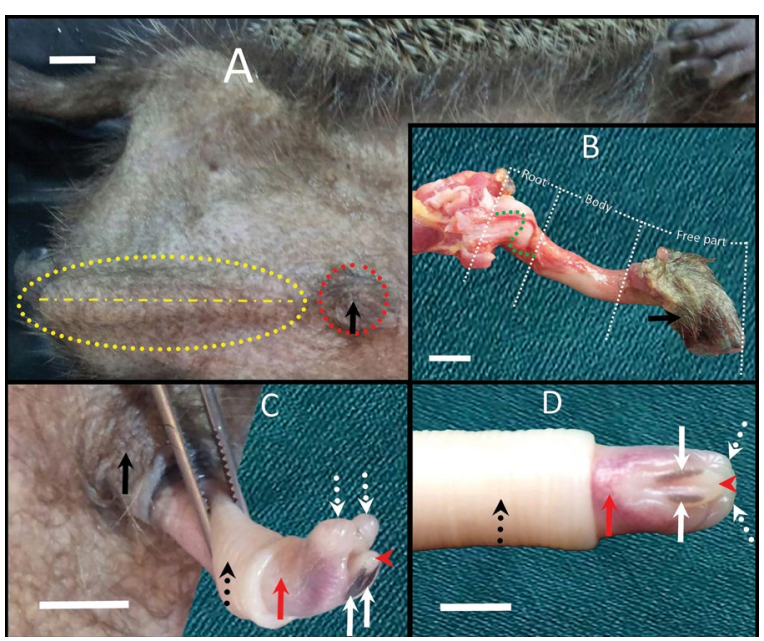

Figure 1. Macroscopic view of the male external genital organ of European hedgehog. Ventral view of the pubic and gastric regions (A). The penis extends from ischiatic arch to umbilical region under the skin (yellow elliptical round dotted line) in cranial direction (yellow dashdotted line). The prepuce is indicated with black arrow $(\mathbf{A}, \mathbf{B}, \mathbf{C})$ in the umbilical region (red round dotted line). At the bulb of the penis, sigmoid flexure (green dash-dotted line) is indicated (B). Finger like glans penis (red arrow) and internal lamina of the prepuce (black round dotted arrow) (C, D). Urethral process (red arrow head) protrudes at the dorsal end of the penis. Sacculus urethralis (white round dotted arrow) situates below to the urethral process with median slit. Penile nails are located dorsally at the end of the penis with white arrow $(\mathbf{C}, \mathbf{D})$. Scale bar: $10 \mathrm{~mm}-\mathrm{A}, \mathrm{B}, \mathrm{C} ; 5 \mathrm{~mm}-\mathrm{D}$.

DP12; Olympus) was used to do the light microscopy and produce images of the histological sections.

\section{RESULTS}

Table 1 shows the mean and standard deviation of the penile length, and penile diameter.

\section{Macroscopic appearance}

The penis of the European hedgehog was connected to the ischiatic arch at the regio urogenitalis with two strong ischiocavernosus muscles (Fig. 2A). The penis 


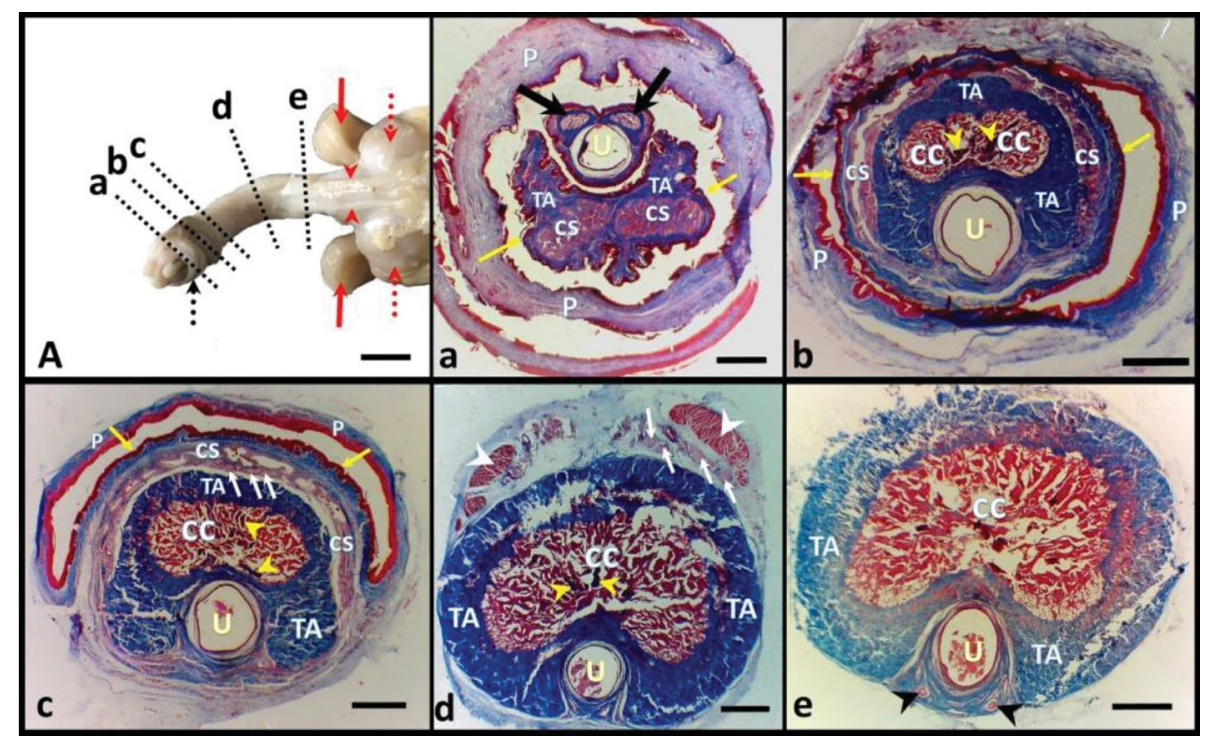

Figure 2. Cross-sections of the European hedgehog penis regions. Dissected macro anatomy of the external genital organ of the European hedgehog (A). The prepuce (black round dotted arrow) is indicated in the free part of the penis. The ischiocavernosus muscles (red round dotted arrow) are located at the bulb of the penis bilaterally and cover the corpus cavernosum. Bulbourethral glands (red arrow) are situated outside the pelvic cavity, on both sides of the anus on the ischiocavernosus muscles. Two retractor muscles (red arrow head) extend to the end of the penile body dorsolaterally. Square dashed lines define the position of each cross-section shown in (a-e). Masson's trichrome staining of the cross-sections of male external genital organ (a-e). In section (a), two penile nails (black arrows) are indicated dorsolaterally to the urethra on each side. The urethra $(\mathrm{U})$ is translocated to the dorsal part of the penis at the end of the glans penis in section (a) but in the sections (b-e) it is located in the ventral part. The prepuce $(\mathrm{P})$ surrounds free part of the penis in sections $(\mathbf{a}-\mathbf{c})$. The corpus spongiosum glans (CS) is located in the dorsal part of penile cross-section and dorsal to the urethra $(\mathbf{b}, \mathbf{c})$ but at the end of the glans penis, it is translocated to the ventral part of the penis and ventral to the urethra and is mostly divided by trabeculae (a). The corpus cavernosum (CC) that exists in sections ( $\mathbf{b}-\mathbf{e})$ is surrounded by tunica albuginea $(T A)(\mathbf{b}-\mathbf{e})$ which sends trabeculae (yellow arrow head) into the corpus cavernosum (b-d), but at the end part of glans penis, the trabeculae become penile nails (a). Two retractor muscles are indicated with white arrow head at the dorsolateral part of penile body cross-section (d). Penile keratinised stratified squamous epithelium is indicated with yellow arrow in sections (a-c). Gatherings of nerve bundles (white arrow) are defined in section (c, d). Two arteries (black arrow head) are indicated ventral to the urethra in section (e). Scale bar: $10 \mathrm{~mm}-\mathrm{A} ; 1000 \mu \mathrm{m}-\mathrm{a}, \mathrm{b}, \mathrm{c}, \mathrm{d}$, e.

directed cranially and extended to the umbilical region (Fig. 1A). This organ, of white colour, with a mean length of $7.17 \pm 0.12 \mathrm{~cm}$, was composed of a pair of corpora cavernosa penis and the glans penis, which was completely covered by the preputium in the non-erection state (Figs. 1, 2A).

The mean length of the corpus penis was $3.58 \pm$ $\pm 0.16 \mathrm{~cm}$ with a mean diameter of $0.83 \pm 0.05 \mathrm{~cm}$. The glans penis in the European hedgehog was red, in the shape of a finger and composed entire of the free part. No collum penis was observed after the glans penis (Fig. 1A).

The penis diameter was the same throughout the body and it decreased a little only in the region of the free part. The length of the free part was a quarter of the total length of the penis. At the beginning of the root, the cavernous bodies were separated from one another and covered with ischiocavernosus muscles (Fig. 2A). In the dorsomedial part of the cavernous bodies and below the rear end of the rectum, the penile bulb, independent of the cavernous bodies, existed and was covered with the bulbocavernosus muscle (Fig. 3A). In this part, a sigmoid flexure was observable (Fig. 1B). After the penile bulb, the urethral diameter decreased and was independently located in the ventral part of the cavernous bodies (Fig. 2e). This structure entered the cavernous bodies at the end of the penile body (Fig. 2b-c). At the end of the penis, it protruded from the dorsal part of the penis as urethral process (Figs. 1B-C, 2a), on both sides of which two black nail-like structures, which were ventrally directed, could be observed (Figs. 1B-C, 2a). The lower part was rounded forming a blind sac (sacculus urethralis) with a median split below the urethra, which tended to widen in the erection state, thereby increasing the urethral opening (Fig. 1B-C).

The retractor penis muscle was very large and extended to the end of the penile body on both dorsolateral sides of the penis (Fig. 2A).

\section{Microscopic appearance}

It was revealed that, in the cross-section of the penile bulb, this region lacked the penile corpus spon- 


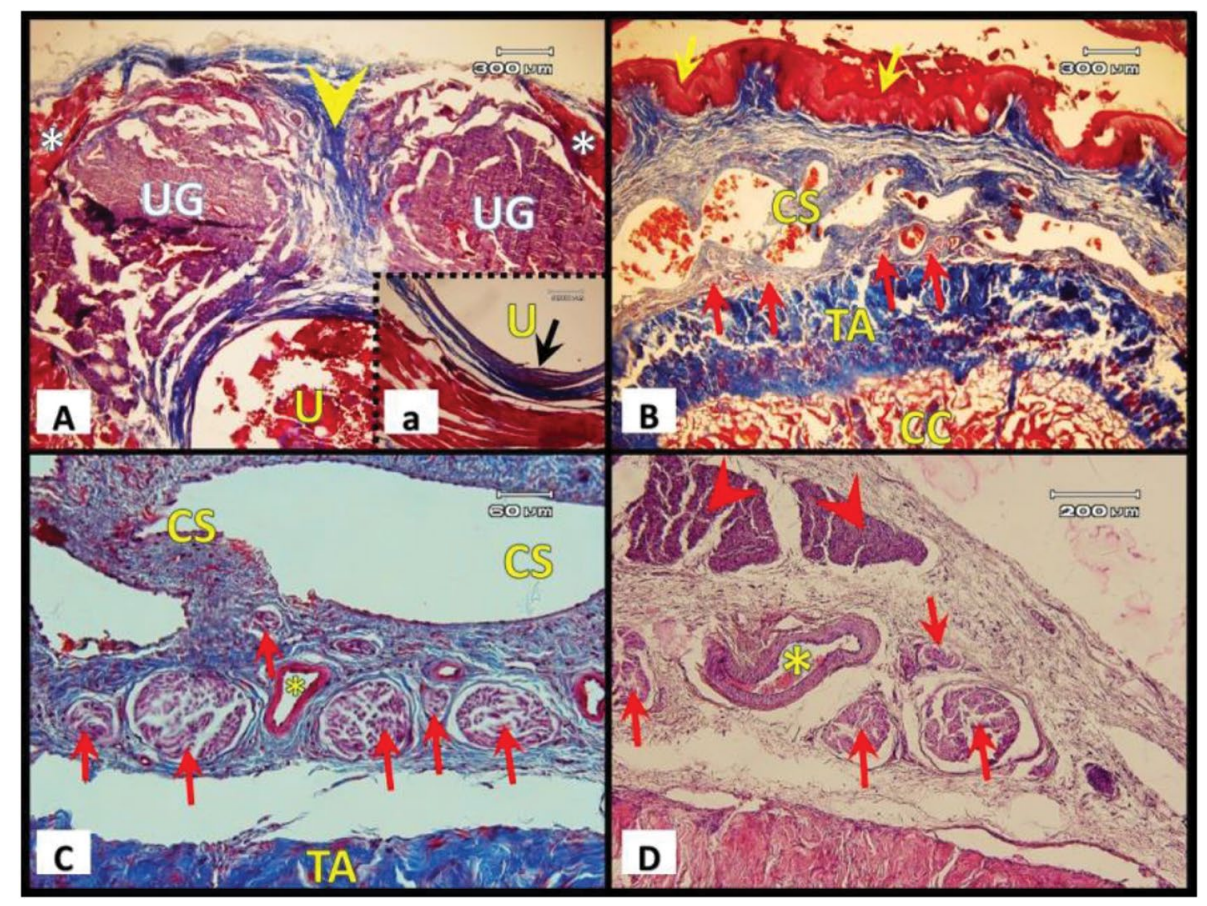

Figure 3. Masson's trichrome (A, a, B, C) and haematoxylin and eosin (D) stained photomicrographs of the European hedgehog penis. Two urethral glands (UG) are located dorsal to the urethra (U) and are separated by trabeculae of tunica albuginea (yellow arrow head); also the bulbospongiosus muscles are shown with white asterisk dorsolaterally (A). The stratified squamous epithelium (black arrow) of the urethra is shown in section (a). The keratinised stratified squamous epithelium of the penis (yellow arrow) is indicated in photograph (B). The corpus spongiosum glans (CS) is shown in sections (B, C). The tunica albuginea (TA) surrounds the corpus cavernosum (CC) (B, C). Lots of nerve fibres (red arrow) are placed between CS and TA (B, C, D). Also, the retractor muscle (red arrow head) is shown in panel D. Supplying arteries (yellow asterisk) are seen in sections (C, D). Scale bar: $300 \mu \mathrm{m}-\mathrm{A}, \mathrm{B} ; 200 \mu \mathrm{m}-\mathrm{a}, \mathrm{D} ; 60 \mu \mathrm{m}-\mathrm{C}$.

giosum. Moreover, in this region, the urethra was very large and circular and had a stratified squamous epithelium (Fig. 3A). In the dorsal part of penile bulb, there were two mucoserous glands separated by the connective tissue. A portion of connective tissue surrounded the urethra as a belt (Fig. $3 A$ ).

It was observed that the tunica albuginea surrounding the corpora cavernosa penis in the hedgehog was composed of the dense regular connective tissue with smooth muscle cells (Fig. 2a-e). Trabeculae entered the cavernous body from the tunica albuginea and were situated among the cavernous bodies (Fig. 2a-e). A small portion of this connective tissue encircled the urethra (Fig. 2a-e). No corpus spongiosum penis was observed around the urethra. The urethral epithelial tissue from the bulbous region to the end was of the stratified squamous type with a large lumen (Fig. 2).

At the beginning of the free part in the dorsolateral region of the cavernous bodies, there was the corpus spongiosum glans. Large veins and lots of nerves were observed inside this part (Figs. 2b, c; $3 B, C)$. Toward the end of the penis, together with the transposition of the urethra to the dorsal part, this structure moved to the ventral part and, at the end of the penis, was located in the ventral region (Fig. 3B). Along with the transposition from the dorsal side to the ventral side, the connective tissue increased in the bodies so that the largest amount of this issue was observed at the end of the penis (Fig. 2a). With the movement of the urethra from the ventral part to the dorsal side, the cavernous bodies grew smaller and were initially separated by the tunica albuginea and, together with the urethra, were pulled up, and, finally, were located on both sides of the urethra as two nails (Fig. 4A, C, D). The movement of the urethra and the cavernous bodies upward created two skin epithelial fold at the end of the penis (Fig. 4A, C).

\section{DISCUSSION}

Used for intercourse, urination, and ejaculation, the external genitals in male mammals provide significant features for their classification.

According to a study by Layne [22], the penile position and shape vary as the sacculus urethralis and 


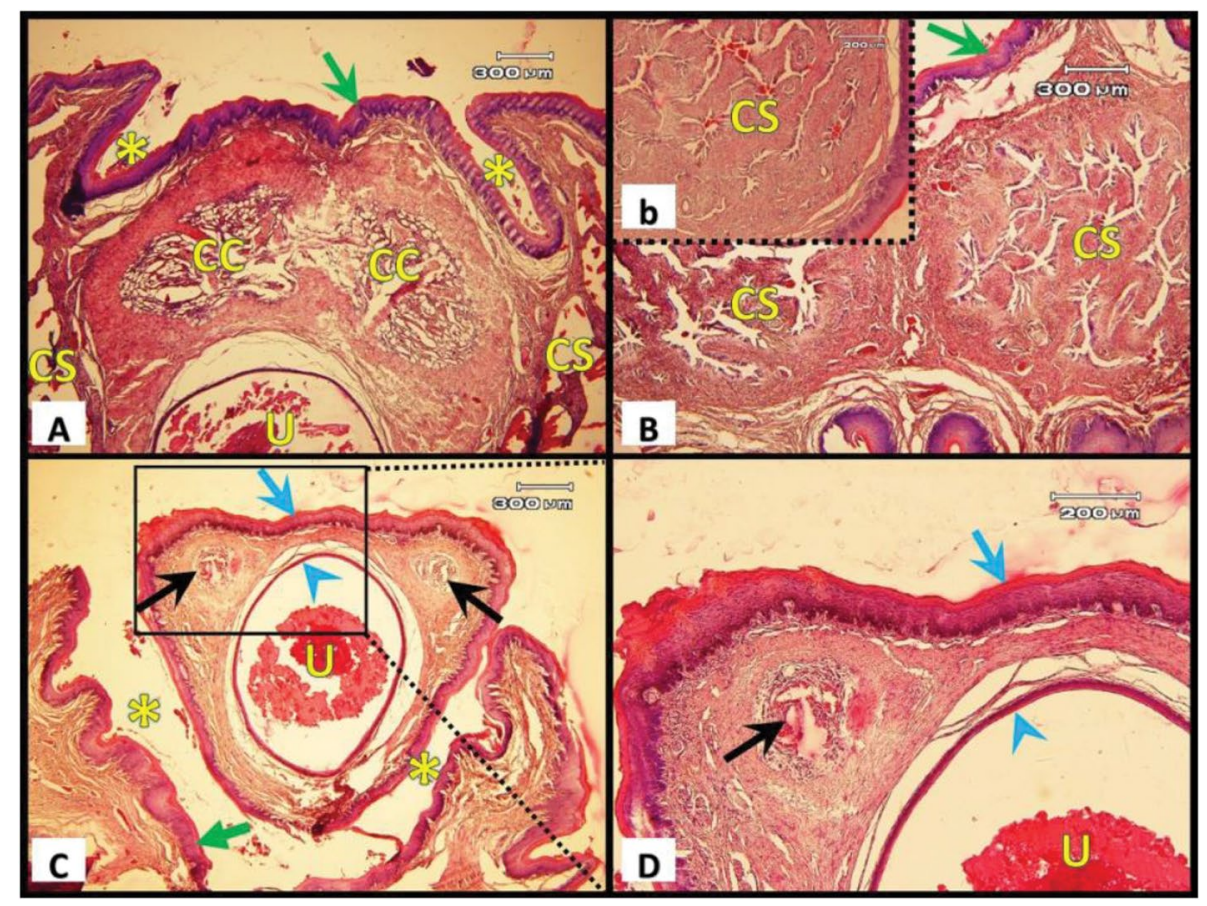

Figure 4. Haematoxylin and eosin stained photographs of the European hedgehog penis. The corpus cavernosum (CC) is located dorsal to the urethra $(\mathbf{U})$ in panel $\mathbf{A}$. The keratinised stratified squamous epithelium of the penis (green arrow) is shown in sections (A, B, C). The corpus spongiosum glans (CS), along dorsal translocation of the urethra, first is placed lateral to the urethra $(\mathbf{A})$ and finally is located ventral to the urethra (B). Panel $\mathbf{b}$ is a higher magnification of corpus spongiosum glans. The movement of the urethra and CC upward created two skin epithelial folds (yellow asterisk) at the end of the penis (A) that urethra accompanied by two penile nails (black arrow) extrude from glans (C). Panel $\mathbf{D}$ is a higher magnification of black rectangular in panel $\mathbf{C}$. The stratified squamous epithelium (blue arrow head) of the urethra, penile nails (black arrows) and the keratinised stratified squamous epithelium of urethral process (blue arrow) are shown in panel $\mathbf{D}$. Scale bar: $300 \mu \mathrm{m}-\mathrm{A}, \mathrm{B}, \mathrm{C} ; 200 \mu \mathrm{m}-\mathrm{b}, \mathrm{D}$.

the os penis in rodents develop. In the present study, the penile morphology of the European hedgehog was explored and compared with other mammals.

According to research results, a retractor penis muscle exists in various species, including boars, horses, dogs, and felines $[3,4,8,14,17]$. These retractor penis muscles are mentioned as smooth muscles in such species but not e.g. in mice or humans. The current study showed that two retractor penis muscles existed as smooth muscles in the European hedgehog. They were situated dorsolaterally to the penis in this animal and extended to the end of the penile body. In this case, European hedgehogs resemble Asian musk shrews (Suncus murinus) [20].

The penis of the European hedgehog is cranially directed and extends to the umbilical region. However, in the rat [18], mouse [12], guinea pig [13, 30], porcupine [5], greater cane rat [1], and Persian squirrel [2], the penis is directed first cranioventrally and then caudoventrally and finally ends up in the glans. Nevertheless, in the elephant shrew, as in the
European hedgehog, the penis is fairly long and extends cranially under the ventral skin and emerges to be caudal to the sternum [37]. The cat is considered the only domestic animal, whose penis is caudally directed [27]. Interestingly, the direction of the penis differs in the European hedgehog and the elephant shrew as compared with other mammals.

The penile bulb in the European hedgehog has a quite unique structure, showing the presence of the sigmoid flexure and the absence of the penile corpus spongiosum. It is composed of two dorsolaterally located seromucinous-glandular tissues, namely urethral glands located in the urethral submucosa and large urethral diverticula. Moreover, the penis is separated from the corpus cavernosum and covered by bulbospongiosus muscles. The presence of the glandular tissues and urethral diverticula has been also reported by Çiner et al. [10] in a study on the male rat and male mouse. However, this research has shown the presence of the penile corpus spongiosum in the male rat and male mouse as well, which is not 
consistent with the findings of the present research on the European hedgehog. In addition, it must be noted that, due to the absence of the penile corpus spongiosum in the European hedgehog, the glandular tissues are located in the urethral submucosa; however, in the male rat and male mouse, these glandular tissues are surrounded by the penile corpus spongiosum [10]. Furthermore, in the Persian squirrel, the cross-section of the penile root indicates that two corpora cavernosa in the root are separated by the wall of the tunica albuginea and united together in the body [2]. Nonetheless, in the penile root and body of the European hedgehog, two corpora cavernosa join into one. The researchers of the study reported that the glandular tissue in the penile bulb was surrounded by the penile corpus spongiosum, which is not in line with the finding about the European hedgehog. Moreover, the lack of the penile corpus spongiosum in the bulb and body of the penis and existence of glans corpus spongiosum which not surrounded the urethra are dissimilar to findings in porcupine [5], male rat and male mouse [10], and Asian musk shrews (Suncus murinus) [20].

According to Tullberg [34], one of the peculiar characteristics of the penis in the suborder Hystricomorpha is the presence of the blind sac in the glans penis ventral to a penile structure called sacculus urethralis, which swells during erection and is in the shape of a balloon. Furthermore, the same structure has been observed in the European hedgehog using a Median Split.

In the distal section of the penis of the European hedgehog, as the urethra is translocated from the ventral to the dorsal part, the corpus cavernosum shrinks and divides by trabeculae coming from the tunica albuginea and extends dorsally along the urethra and finally appears as two nail-like, needle-shaped keratinaceous structures on both sides of the urethral process. These structures have a central cavity which proves that these nails are the elongation of the corpus cavernosum. In addition, these structures also exist in the guinea pig [13], porcupine [5], and agouti [25]. Despite this, it must be noted that, in these animals, unlike the European hedgehog, the nail-like structures are not the continuance of the corpus cavernosum. According to Atalar and Ceribasi [5], these are penile nail-like structures, which other animals lack. This could be regarded as a secondary sexual characteristic. Moreover, these penile nail-like structures bend downward in European hedgehogs.
They possibly play the role of an anchorage for the penis during the penetration into the vagina in copulation. Furthermore, they might provide more sexual stimulation. Akbari et al. [2] reported the presence of this structure in the Persian squirrel; however, that is an individual structure and consists of the hyaline cartilage that overextends from the penile tip; moreover, it lacks the central cavity.

In some mammals, for example, the cat [27], African mole rat [29], porcupine [5], guinea pig [13], agouti [25], and mouse [26], there are penile spines or cornified papillae on the body and the glans penis. Nonetheless, in the European hedgehog, as in the Persian squirrel [2], these spines are not observable. Moreover, the presence of these papillae can be regarded as a secondary sexual feature as well [5].

Wrobel and Bergmann [38] classified the penis into three types according to the development of erectile rather than connective tissues. The first one, "vascular-type penis," contained bundles of erectile tissues. The second one, "fibrous-type penis," had well-developed connective tissues. The third one, "intermediate-type penis," had moderate erectile and connective tissues. Based on this classification, the type of the penis is vascular in humans and equidae and it is fibrous in ruminants and boars [6]. In addition, the intermediate type is seen in the dog and cat [38]. According to the results of this research, the moderate erectile and connective tissues were observed in the penis of the European hedgehog, which classified it as the intermediate-type penis. Furthermore, Atalar and Ceribasi [5] stated that the type of the penis in the rat, mouse, and porcupine resembled that in carnivores. Moreover, Akbari et al. [2] categorised the penis of the Persian squirrel as the intermediate type, which is the case with the penis of the European hedgehog. Moreover, Adebayo et al. [1] classified the penis of the greater cane rat as the intermediate type.

According to a research study, carnivores have a grooved catheter-shaped os penis at the penile body with the middle part in the cross-section of the penis, which is the cartilaginous structure at the penile glans [27]. However, in the rat and mouse, that the os penis is seen in the proximal and distal portions of the glans penis $[26,31,35,36,39]$. In the Japanese macaca, it has only a proximal segment [40]. Interestingly, like carnivores, porcupines have an os penis in the form of a grooved catheter and a glans penis that has the cartilaginous structure of the os penis. In spite of this, dif- 
ferent from carnivores, the os penis is situated in the dorsal part of the transversal section of the penis [5]. Furthermore, Mollineau et al. [25] declared that the os penis in the agouti had a racket shape, was situated dorsally in the cross-section, and had two cartilaginous structures on both sides. Additionally, in the Persian squirrel, such a structure is in the shape of a cone and extends from the middle part of the glans penis to the distal part [2]. In contrast with above-mentioned points, in the European hedgehog, no os penis or cartilaginous structure was observed.

According to some researchers [6, 33], from inside to outside, the penile structure of domestic and laboratory animals is composed of the internal lamina of preputium, penile epidermis, tunica albuginea, i.e. fibrous-type penis, penile corpus cavernosum, penile corpus spongiosum, urethral sulcus, and urethra. All of these exist in the Persian squirrel [2] though tunica albuginea does not exist in the porcupine [5]. Elastic and collagen fibres are significant penile structures in erection state and develop sufficient resistance to return to the non-erection state [7]. In the hedgehog, all of these structures, except for the corpus spongiosum, exist.

Maia et al. [23] reported that elastic fibres rate in rabbit penis was intense and classified the type of the penis in this animal as the vascular type. Also, elastic fibres rate in Persian squirrel is intense and collagen fibres rate in corpus cavernosum and tunica albuginea is remarkable [2]. According to the findings of the present study on the European hedgehog, the rate of elastic and collagen fibres is higher than that of the corpus spongiosum.

Furthermore, noticeably, a large number of the cavernous tissues are alternated by connective tissues while moving from the corpus to the glans penis.

As for the penile structure, it can be said that the penile body and the glans penis have a distinguishable border as collum penis. Nevertheless, in the European hedgehog, this obvious border is not observable. However, in equidae, carnivores [16], rabbits [30] and porcupines [5], the penile body and the glans penis have a distinguishable border. In addition, according to Atalar and Ceribasi [5], the colour of the glans penis in porcupines is black, and the corpus penis is white. This can help us determine the borders of the two parts. Moreover, in the greater cane rat [1], as in the European hedgehog, the collum penis does not exist.

The penile urethra in the European hedgehog lined by stratified squamous epithelium from the bulbous region to the end. But, Atalar and Ceribasi [5] on the porcupine and Tanyolac [33] report that the penile urethra is lined by a transitional epithelium, which was transformed into a stratified squamous epithelium toward the tip of the glans penis which is dissimilar to our findings in European hedgehog.

\section{CONCLUSIONS}

In conclusion, it must be remarked that the penis of the European hedgehog was classified as the intermediate-type penis. In addition, the existence of penile nails with a central cavity is one of the secondary sexual characteristics, probably acting as an anchorage for the penis during penetration into the vagina in copulation. Moreover, the existence of sigmoid flexure in the penile bulb may be of significance. Also, absence of penile corpus spongiosum at the bulb of the penis and body of it can be mentioned as a specific feather different compared with other mammals; but on the other hand, the urethra in the glans penis is not surrounded by corpus spongiosum glans and, the entire penile urethra is not surrounded by corpus spongiosum. In the end, it should be pointed out that thoroughly stratified squamous epithelium of penile urethra and bilateral urethral glands in the European hedgehog are basically different from those in other mammals.

The present research is the first report on the anatomy of the penis in the European hedgehog and will surely contribute to this field.

\section{REFERENCES}

1. Adebayo AO, Akinloye $A K$, Olurode $S A$, et al. The structure of the penis with the associated baculum in the male greater cane rat (Thryonomys swinderianus). Folia Morphol. 2011; 70: 197-203.

2. Akbari G, Adibmoradi M, Gilanpour H, et al. Anatomical and histological study of penis in Persian squirrel (Sciurus anomalus). J Vet Res. 2014; 69: 401-409.

3. Aronson LR, Cooper ML. Seasonal variation in mating behavior in cats after desensitization of glans penis. Science. 1966; 152(3719): 226-230, indexed in Pubmed: 12325355.

4. Ashdown RR, Barnett SW. Impotence in the boar: angioarchitecture and venous drainage of the penis in normal boars. Vet Rec. 1981; 109(17): 375-382, indexed in Pubmed: 7200281.

5. Atalar O, Ceribasi AO. The morphology of the penis in porcupine (Hystrix cristata). Vet Med. 2018; 51(No. 2): 66-70, doi: 10.17221/5520-vetmed.

6. Banks WJ. Applied Veterinary Histology. Male reproductive system, 2nd ed. Williams \& Wilkins, London 1986: 502-505.

7. Bastos AL, Silva EA, Silva Costa W, et al. The concentration of elastic fibres in the male urethra during human fetal development. BJU Int. 2004; 94(4): 620-623, doi: 10.1111/j.1464410X.2004.05012.x, indexed in Pubmed: 15329125. 
8. Bo Minelli L, Acone F, Zedda M, et al. Further observations on the morphology and sensitive innervation of the retractor penis muscle in several species of ungulates. Ital J Anat Embryol. 1993; 98(1): 13-21, indexed in Pubmed: 8343009.

9. Bradley RD, Schmidly DJ, Owen RD. Variation in the Glans Penes and Bacula among Latin American Populations of the Peromyscus boylii Species Complex. J Mammal. 1989; 70(4): 712-725, doi: 10.2307/1381706.

10. Ciner M, van Vorstenbosch CJ, Dijkstra G, et al. Penile bulb and its relationship with the pelvic urethra and the penile urethra in the rat: light and scanning electron microscopical observations. Anat Rec. 1996; 244(4): 452-469, doi: 10.1002/ (SICI)1097-0185(199604)244:4<452::AID-AR4>3.0.CO;2-X, indexed in Pubmed: 8694281.

11. Colbert EH. Evolution of the Vertebrates. Wiley, New York. 1958.

12. Cook MJ. The anatomy of the laboratory mouse. Academic Press, London, New York. 1965.

13. Cooper G, Schiller AL. Anatomy of the Guinea Pig. Harvard University Press, Cambridge, MA. 1975.

14. Cunha GR, Wang Y, Place NJ, et al. Urogenital system of the spotted hyena (Crocuta crocuta Erxleben): a functional histological study. J Morphol. 2003; 256(2): 205-218, doi: 10.1002/jmor.10085, indexed in Pubmed: 12635111.

15. Dinc G, Yilmaz S, Girgin A, et al. A light microscopic study on the of penis in rat. Firat University Journal of Health Sciences. 1996; 10: 149-152.

16. Dursun N. Veterinary Anatomy II. Penis (in Turkish). Medisan Yayinevi, Ankara. 1996: 153-159.

17. Fisher $\mathrm{H}$. Histological structure of the retractor penis muscle of the dog. Anat Rec. 1917; 13(2): 69-79, doi: 10.1002/ ar.1090130202.

18. Green EC. Anatomy of the rat. Transaction of the American Philosophical Society Held at Philadelphia for promoting useful knowledge. Vol. XXVII. Hafner Publishing Company, New York. 1963: 117-121.

19. Gultiken ME, Yildiz D, Bolat D. The anatomy of the red fox (Vulpes vulpes). Vet J Ankara Univ. 2004; 51: 71-73.

20. Kamikawa-Miyado M, Ogi H, Ogino Y, et al. The morphological and histological characters of the male external genitalia of the house musk shrew, Suncus murinus. Zoolog Sci. 2005; 22(4): 463-468, doi: 10.2108/zsj.22.463, indexed in Pubmed: 15846055.

21. Kondo $S$, Hanamura $H$, Ishikawa $A$, et al. An odontometrical difference in the mandibular molars of two laboratory strains of the musk shrew, Suncus murinus, derived from Bangladesh and Tokunoshima Island of Japan. Okajimas Folia Anat Jpn. 1999; 76(5): 231-239, indexed in Pubmed: 10693326.

22. Layne JN. The glans penis and baculum of the rodent Dactylomys dactylinus Desmarest. Mammalia. 2009; 24: 87-92.

23. Maia RS, Babinski MA, Figueiredo MA, et al. Concentration of elastic system fibers in the corpus cavernosum, corpus spongiosum, and tunica albuginea in the rabbit penis. Int J Impot Res. 2006; 18(2): 121-125, doi: 10.1038/sj.ijir.3901404, indexed in Pubmed: 16224492.

24. McLaughlin CA, Chiasson RB. Laboratory anatomy of the rabbit. Urinary and reproductive system. 2 nd ed. W.C. Brown Company, lowa. 1979: 80-86.
25. Mollineau W, Adogwa A, Jasper N, et al. The gross anatomy of the male reproductive system of a neotropical rodent: the agouti (Dasyprota leporina). Anat Histol Embryol. 2006; 35(1): 47-52, doi: 10.1111/j.1439-0264.2005.00656.x, indexed in Pubmed: 16433673.

26. Murakami R. A histological study of the development of the penis of wild-type and androgen-insensitive mice. J Anat. 1987; 153: 223-231, indexed in Pubmed: 3429322.

27. Nickel R, Schummer A, Seiferle E. The Anatomy of the domestic animals. Male genital organs. Vol. 2. Verlag Paul Parey, Berlin. 1981: 304-348.

28. Novacek MJ. Mammalian phylogeny: shaking the tree. Nature. 1992; 356(6365): 121-125, doi: 10.1038/356121a0, indexed in Pubmed: 1545862.

29. Parag A, Bennett NC, Faulkes CG, et al. Penile morphology of African mole rats (Bathyergidae): structural modification in relation to mode of ovulation and degree of sociality. J Zool. 2006; 270(2): 323-329, doi: 10.1111/j.14697998.2006.00141.x.

30. Popesko P, Rajtov V, Horak J. Colour atlas of the anatomy of small laboratory animals. Vol. 1. Wolfe Publishing Ltd. London. 1990: 119-222.

31. Rasmussen KK, Vilmann $\mathrm{H}$, Juhl M. Os penis of the rat. V. The distal cartilage process. Acta Anat (Basel). 1986; 125(3): 208-212, indexed in Pubmed: 3962582.

32. Simson S, Ferrucci L, Kurtonur C, et al. Phalli and Bacula of European Dormice: description and comparison. HYSTRIX, the Italian Journal of Mammalogy. 1995; 6: 231-244, doi: 10.4404/hystrix-6.1-2-4035.

33. Tanyolac A. Ozel Histoloji. Penis. Yorum Basin Yayin Ltd. Ankara. 1993: 139-141.

34. Tullberg T. Ueber das System der Nagethiere: eine phylogenetische Studie. Akademische Buchdruckerei. 1899.

35. Vilmann A, Vilmann H. Os penis of the rat. IV. The proximal growth cartilage. Acta Anat (Basel). 1983; 117(2): 136-144, doi: 10.1159/000145779, indexed in Pubmed: 6685422.

36. Vilmann $\mathrm{H}$. Os penis of the rat. III. Formation and growth of the bone. Acta Morphol Neerl Scand. 1982; 20(4): 309-318, indexed in Pubmed: 7158443.

37. Woodall PF. The penis of elephant shrews (Mammalia: Macroscelididae). J Zool. 1995; 237(3): 399-410, doi: 10.1111/ j.1469-7998.1995.tb02770.x.

38. Wrobel KH, Bergmann M. Male Reproductive System. In: Eurell JA, Frappier BL (eds.). Dellman's textbook of histology. 6th ed. Blackwell Publishing Ltd. lowa. 2006: 233-255.

39. Yamamoto M, Umekita S, Nishimura M. Studies on the os penis of the rat and Japanese macaca. Teikyo Medical J. 1990; 13: 375-383.

40. Yamamoto M. Histology of the os penis of the rat. I. Light microscopic study of the distal segment and possible origin of its osteocytes. Teikyo Medical J. 1987; 10: 303-313.

41. Yildiz D, Bolat D, Karahan S. The Morphology of the Os Penis in the Adult Mouse. J Anim Vet Adv. 2010; 9(14): 1913-1917, doi: 10.3923/javaa.2010.1913.1917.

42. Youssefi MR, Rahimi MT, Halajian A, et al. Helminth Parasites of Eastern European Hedgehog (Erinaceus concolor) in Northern Iran. Iran J Parasitol. 2013; 8(4): 645-650, indexed in Pubmed: 25516749. 